\title{
Intrinsic emittance reduction of copper cathodes by laser wavelength tuning in an rf photoinjector
}

\author{
Marta Csatari Divall, ${ }^{1,}$ Eduard Prat, ${ }^{1}$ Simona Bettoni, ${ }^{1}$ Carlo Vicario, ${ }^{1}$ Alexandre Trisorio, ${ }^{1}$ \\ Thomas Schietinger, ${ }^{1}$ and Christoph P. Hauri ${ }^{1,2}$ \\ ${ }^{1}$ Paul Scherrer Institut, 5232 Villigen PSI, Switzerland \\ ${ }^{2}$ Ecole Polytechnique Fédérale de Lausanne, 1015 Lausanne, Switzerland
}

(Received 26 March 2014; published 30 March 2015)

\begin{abstract}
With the improvement of acceleration techniques, the intrinsic emittance of the cathode has a strong impact on the final brightness of a free electron laser. The systematic studies presented in this paper demonstrate for the first time in a radiofrequency photocathode gun a reduction of the intrinsic emittance when tuning the laser photon energies close to the effective work function of copper. The intrinsic emittance was determined by measuring the core slice emittance as a function of the laser beam size at laser wavelengths between 260 and $275 \mathrm{~nm}$. The results are consistent with the measured effective work function of the cathode. Slice emittance values normalized to the laser beam size reached values down to $500 \mathrm{~nm} / \mathrm{mm}$, close to that expected from theory. A $20 \%$ reduction of the intrinsic emittance was observed over the spectral range of the laser.
\end{abstract}

DOI: 10.1103/PhysRevSTAB.18.033401

PACS numbers: 07.77.Ka, 42.62.-b, 79.60.-i, 81.05.Bx

\section{INTRODUCTION}

The recent advent of 4th generation free electron lasers (FELs) delivering femtosecond $\mathrm{x}$-ray pulses at intensities beyond $10^{14} \mathrm{~W} / \mathrm{cm}^{2}$ has opened new opportunities in exploring ultrafast molecular and atomic phenomena [1-3]. Understanding the interaction of such intense ultrashort $\mathrm{x}$-ray pulses with matter will have an impact on a wide range of scientific disciplines including biology, chemistry and condensed matter physics. As the studies of multielectron dynamics evolve on a femtosecond or even attosecond time scale, ultrashort $\mathrm{x}$-ray pulses are an essential tool to visualize and control the collective electronic rearrangements and atomic displacements. Beyond the current FELs future light sources are aiming for higher repetition rates and reduced machine length and cost. Applications, such as ultrafast, single-shot, single-molecule diffraction imaging $[4,5]$ call for ultrashort, bright $\mathrm{x}$-ray pulses at high repetition rate. To meet these demands a growing number of compact FELs are being developed with optimized, low emittance electron beams [6-9], including SwissFEL at the Paul Scherrer Institute (PSI) [10]. The SwissFEL facility will produce coherent, bright, short $\mathrm{x}$-ray pulses covering the wavelength range from $1 \AA$ up to $70 \AA$.

State of the art high brightness accelerator facilities have been optimized to minimize the start-to-end emittance

\footnotetext{
"marta.divall@psi.ch
}

Published by the American Physical Society under the terms of the Creative Commons Attribution 3.0 License. Further distribution of this work must maintain attribution to the author $(s)$ and the published article's title, journal citation, and DOI. growth to such an extent that the electron source becomes a significant contributor to the final emittance and brightness of the electron beam. The intrinsic emittance, which is proportional to the transverse momentum of the electrons exiting the cathode, is related to the electrons' initial kinetic energy. Transversely coherent FEL radiation is generated efficiently when the electron beam overlaps the FEL radiation along the undulator line. This condition can be expressed in first approximation as $\varepsilon_{n} / \gamma<\lambda / 4 \pi$, where $\varepsilon_{n}$ is the normalized beam emittance, $\gamma$ is the Lorentz factor and $\lambda$ is the FEL radiation wavelength. This condition indicates that the final beam energy can be decreased only by reducing the emittance at the undulator line, which translates into a more compact and cost-effective accelerator. At a given energy, a smaller intrinsic emittance implies a higher radiation power and a shorter undulator beam line to reach FEL saturation.

To illustrate the effect of the intrinsic emittance on the final FEL performance, Fig. 1 shows the dependence of the FEL power on the intrinsic emittance for the $200 \mathrm{pC}$ charge operation mode of SwissFEL. We optimized the normalized emittance along the bunch at the exit of the injector as a function of the intrinsic emittance, taking the SwissFEL nominal laser pulse length of 9.9 ps FWHM. An optimizer [11] automatically determines the best working point for the peak field of the solenoid surrounding the gun and the transverse laser beam size, while iteratively running the numerical simulation code AsTRA [12]. We assume that the normalized emittance is preserved from the injector exit to the undulator entrance, where the beam energy is $5.8 \mathrm{GeV}$. For each obtained emittance value at the undulator line the FEL power for a radiation wavelength of $1 \AA$, after eight undulator modules, is then calculated with the 


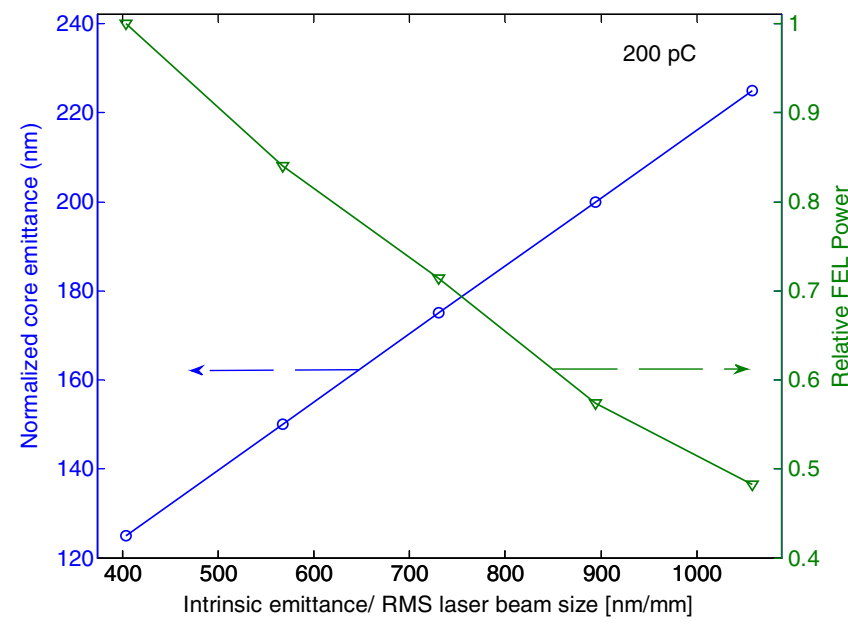

FIG. 1. Dependence of the relative FEL power as a function of the intrinsic emittance. Normalized core slice emittance is simulated for the $200 \mathrm{pC}$ charge operation mode of SwissFEL for different normalized intrinsic emittance values. The corresponding normalized emittance at the undulator entrance for the best working points (dots) is then used to estimate the relative FEL power (triangles).

code GENESIS [13]. We assume the other beam parameters to be the design ones for SwissFEL at $200 \mathrm{pC}$, thus the variation in FEL power is solely associated with the change in brightness. We note that for the simulated cases the FEL reaches saturation within eight undulator modules. For instance, the FEL power increases by about $25 \%$ for an intrinsic emittance reduction from 600 to $400 \mathrm{~nm} / \mathrm{mm}$.

For low charge beams where space-charge effects become negligible, the final emittance is mostly determined by the intrinsic emittance. At low charge, short electron pulses with ultralow emittance can be generated, producing subfemtosecond narrow band single FEL spikes $[6,14,15]$. Low charge in turn also requires less laser energy and hence opens up the possibility for higher repetition rates. Therefore a reduction of the intrinsic emittance of the electron source has a direct impact on future developments and potentially on the performance of existing x-ray FEL sources.

The optimization of the transverse and the longitudinal profiles of the laser beam aiming to improve projected and slice emittance in photoinjectors has been intensively investigated $[7,9,16,17,18]$. In this work, performed at the SwissFEL Injector Test Facility (SITF) [19], we investigate experimentally for the first time in a radiofrequency (rf) gun the tunability of the intrinsic emittance as a function of the laser wavelength. The intrinsic emittance is close to the theoretical predictions when using the effective work functions determined experimentally in SITF and in excellent agreement with theoretical expectations [20].

\section{INTRINSIC EMITTANCE}

The laser wavelength is usually chosen to slightly exceed the natural work function of the cathode within the boundaries of available laser sources. The photoemission model applied to rf guns [20] shows that the intrinsic emittance can be reduced by selecting photon energies closer to the effective work function at the cost of reduced quantum efficiency (QE). The theory was supported experimentally by wavelength tuning in a dc gun [21], while measurements in a $100 \mathrm{MV} / \mathrm{m}$ rf gun have reported ratios of intrinsic emittance versus laser beam size of $\sim 900 \mathrm{~nm} / \mathrm{mm}$, which is about 2 times higher than expected for copper cathodes at $250 \mathrm{~nm}$ laser wavelength $[6,22]$.

The contributions to the intrinsic emittance are given by

$$
\varepsilon_{\mathrm{int}}=\sigma_{l} \sqrt{\frac{\varphi_{l}-\varphi_{\mathrm{eff}}}{3 m_{e} c^{2}}}
$$

where $\varphi_{l}$ is the laser photon energy in $\mathrm{eV}, \sigma_{l}$ is the rms laser beam size, $m_{e} c^{2}$ is the electron's rest mass energy and $\varphi_{\text {eff }}$ is the effective work function. We will call the second term of Eq. (1) normalized intrinsic emittance, expressed in $\mathrm{nm} / \mathrm{mm}$, as it is independent of the laser beam size. The effective work function is defined as the material work function $\varphi_{w}$, reduced by the Schottky effect $\varphi_{\text {Sch }}$ [23-25]:

$$
\begin{gathered}
\varphi_{\mathrm{eff}}=\varphi_{w}-\varphi_{\mathrm{Sch}}, \\
\varphi_{\mathrm{Sch}}=\sqrt{\frac{e^{3}}{4 \pi \varepsilon_{0}} \beta E_{\mathrm{cath}} \sin \phi,}
\end{gathered}
$$

where $\varepsilon_{0}$ is the vacuum permittivity, $e$ is the charge of the electron, $E_{\text {cath }}$ is the maximum rf field at the cathode surface and $\phi$ is the operational rf phase. Here we have added an enhancement factor $\beta$, proposed in Refs. [22,25,26], which includes all surface related effects.

The quantum efficiency is linked to the effective work function $[20,22]$ :

$$
Q E \propto\left(\varphi_{l}-\varphi_{\mathrm{eff}}\right)^{2} .
$$

This relation allows the determination of the effective work function, in the space-charge-free regime, by measuring the dependence of the QE on the photon energy or on the external field.

A purely theoretical estimation of the intrinsic emittance is difficult. For the work function of copper, values in the literature range from 4.3 [27] to $4.6 \mathrm{eV}$ [28]. This value is influenced by crystal structure, surface roughness, impurities and contamination and, as a result, varies from cathode to cathode. In an rf gun the potential barrier is lowered by the externally applied high electric field. Roughness of the cathode with sharp peaks will result in increased QE as well as an enhancement of the Schottky effect and hence an increase of the intrinsic emittance $[20,22,26,29]$. Other surface effects can deteriorate the intrinsic emittance due to larger transverse momentum, without affecting the QE. Earlier works mentioned above 
have taken the theoretical values for intrinsic emittance using the Schottky effect without considering possible enhancement effects. An increased Schottky effect results in a lower effective work function and hence higher intrinsic emittance. Although rf fields can be measured with high accuracy, the direct evaluation of cathode surface effects is difficult. The reported effective work function values therefore vary by up to $\sim 1 \mathrm{eV}$ for an equivalent field at the cathode $(\sim 50 \mathrm{MV} / \mathrm{m})[18,25]$. In summary, complex cathode surface effects spoil the clear correlation between intrinsic emittance and QE. To overcome these issues we have determined the effective work function of the employed cathode directly from measurements in the rf gun in two independent ways: first by measuring the emitted charge as a function of the laser photon energy [based on Eq. (4)] and second as a function of the injection phase [Schottky scan based on Eqs. (2)-(4)]. In addition, when the measurements are performed with sufficiently low charge to avoid spacecharge effects, the emittance over the central longitudinal slice of the bunch (core slice emittance) at the gun corresponds to the intrinsic emittance. We compare the indirect intrinsic emittance values from the work function to the direct core slice emittance measurements at low charge.

\section{THE SWISSFEL INJECTOR TEST FACILITY}

The SwissFEL Injector Test Facility (SITF) was a $250 \mathrm{MeV}$ injector aiming to demonstrate the high-brightness electron beam required to drive the SwissFEL accelerator [19]. It was in operation between 2010 and 2014.

The photocathodes were mounted in a load-lock chamber and inserted in an S-Band 2.5-cell normal conducting gun (CTF gun [30]) operating at $2.997 \mathrm{GHz}$ with a peak onaxis accelerating gradient of $100 \mathrm{MV} / \mathrm{m}$ to keep spacecharge effects under control. Because of the geometry of the cavity backplane this corresponds to $84.23 \mathrm{MV} / \mathrm{m}$ peak field on the cathode. Two symmetric rf coupling ports are connected to the left and right side of the central cell, canceling the dipole component of the rf field but without correction of the resulting quadrupole component.

The cathode used for our measurements is labeled $\mathrm{Cu}_{-} 3$, prepared from $\mathrm{Cu}-\mathrm{OFE}$ material and diamond turned. Before installation into the load-lock chamber the cathode was cleaned in an ultrasound bath of acetone and alcohol and baked for 10 hours at $250^{\circ} \mathrm{C}$. The cathode was installed in February 2012. The QE, measured with the smallest available laser beam size ( $50 \mu \mathrm{m} \mathrm{rms})$, varied by about $15 \%$ across the area of measurements $( \pm 1 \mathrm{~mm})$ shown here. The initial QE at $262 \mathrm{~nm}$ was $0.01 \%$.

The photoemission is achieved with a large-bandwidth high-power Ti:sapphire laser system [31]. The system allows the tuning of the central wavelength around $800 \mathrm{~nm}$ giving a 3rd harmonic emission between 260 and $280 \mathrm{~nm}$. This was further extended to $250-310 \mathrm{~nm}$ by the use of an optical parametric amplifier (OPA) and a conversion stage [32]. The OPA was only used to perform
QE measurements, the amplitude and beam profile stability with the OPA were not satisfactory for reliable intrinsic emittance measurements. Furthermore a directly diode pumped Nd:YLF laser system was available at a fixed 4thharmonic wavelength of $262 \mathrm{~nm}$. A longitudinal profile of 10 ps FWHM was selected for the measurements presented here and circular apertures were used to cut the central homogeneous part of the laser beam, which was imaged onto the cathode for each wavelength at near to normal incidence. The laser beam size was varied by changing the aperture. The laser beam was aligned on the rf axis of the gun using the image of the dark current as a reference.

\section{INTRINSIC EMITTANCE MEASUREMENTS}

Slice emittance measurements were performed by vertically deflecting the beam onto a high-resolution yttriumaluminum-garnet (YAG) screen based on a novel imaging geometry [33] in the high-energy section $(250 \mathrm{MeV})$ of the accelerator, correlating the longitudinal bunch slices to the vertical positions on the screen. For the emittance reconstruction the transverse beam size of each deflected slice is measured at different phase advances of the transfer optics. A detailed description of the core slice emittance measurements can be found in Ref. [8].

To determine the core slice emittance, we divide the bunch into ten slices per rms bunch length and average the emittance over five slices around the longitudinal beam center. The error associated to the core slice emittance measurement considers the variation of the emittance along these slices and the statistical uncertainty of each individual emittance measurement.

In this section we present our results on the cathode intrinsic emittances obtained from both core slice emittance measurements at low charge and estimates of the effective work functions from $\mathrm{QE}$ scans in the rf gun. For the former, we first demonstrate the absence of space-charge effects in the slice emittance measurements.

\section{A. Space-charge effects}

The novel YAG screen has made possible measurements at less than $1 \mathrm{pC}$, where space-charge effects are negligible. This was confirmed by reducing the charge at fixed laser beam size, until the emittance was no longer reduced, as shown in Fig. 2. Measurements were performed at $1 \mathrm{pC}$ bunch charge, corresponding to $\sim 25 \mathrm{pC} / \mathrm{mm}^{2}$ surface charge density, while applying a $\sim 50 \mathrm{MV} / \mathrm{m}$ field on the cathode. The laser pulse length was 10 ps FWHM.

\section{B. Laser beam size scans}

In Fig. 3 we show the core slice emittance measurements as a function of laser beam size, for two different wavelengths. The charge surface density was kept constant during the measurements to avoid space-charge effects. A core slice emittance as small as $30 \mathrm{~nm}$ could be measured [Fig. 3(a)] by applying the smallest aperture, corresponding 


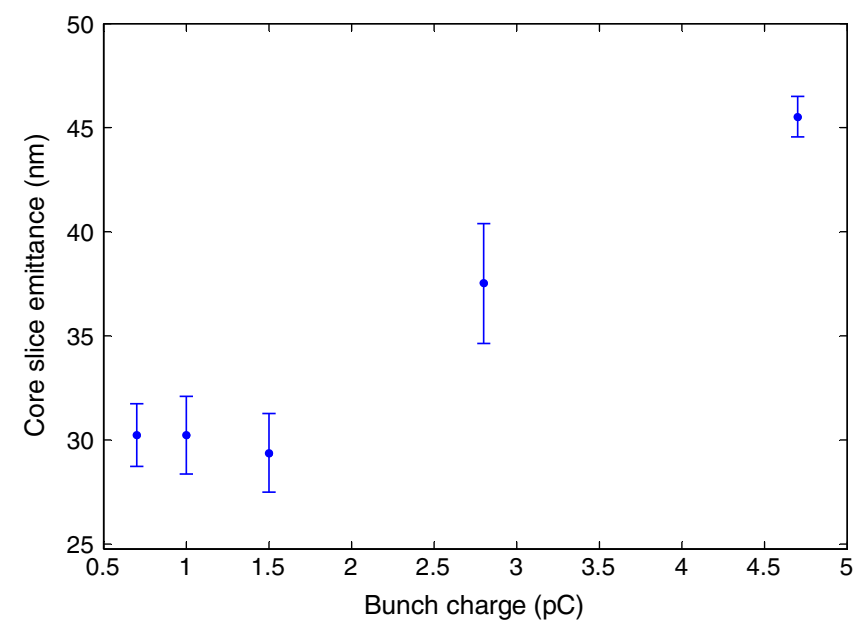

FIG. 2. Measured slice emittance as a function of bunch charge at $262 \mathrm{~nm}$ laser wavelength with $50 \mu \mathrm{m}$ rms beam size on the cathode to determine the space-charge limit. Measurements at less than $1.5 \mathrm{pC}$ charge give the same core slice emittance values within the accuracy of the measurement, while at higher charge the presence of space-charge effects becomes clear.

to $50 \mu \mathrm{m}$ rms laser beam size at the cathode. For increasing beam size we observed a quasilinear growth of the normalized intrinsic emittance [Fig. 3(b)].

This is in contradiction with Eq. (1), but it can be explained by the CTF gun design, which exposes the electron beam to a quadrupole field component induced by the noncompensated two-port rf coupling geometry [34]. We measured core slice emittance at the smallest aperture while moving the laser beam across the cathode: the slice emittance variation was within the measurement error, so the inhomogeneity of the cathode was ruled out as an
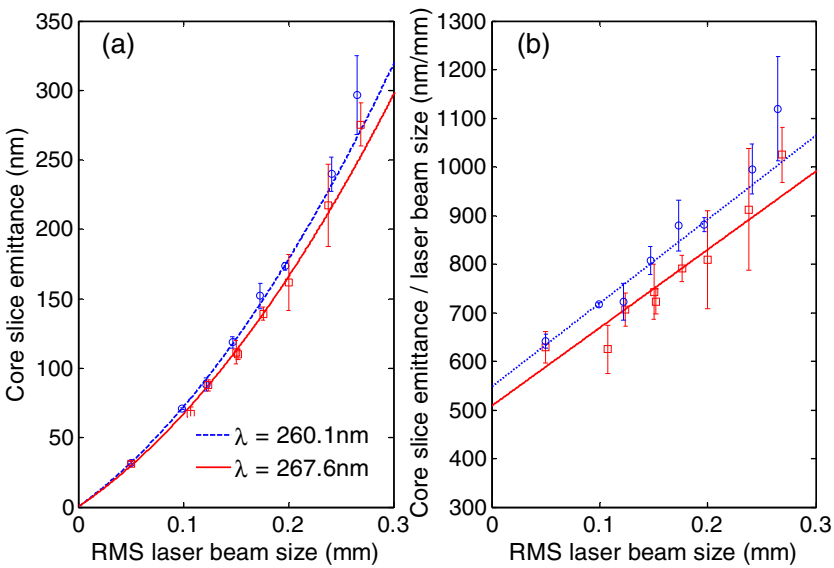

FIG. 3. (a) Core slice emittance as a function of the laser beam size for two laser wavelengths - $260.1 \mathrm{~nm}$ (dashed blue line) and $267.6 \mathrm{~nm}$ (red solid line)_for cathode $\mathrm{Cu}_{-} 3$. The second order fit, taking into account the quadrupole component in the rf field, gives a linear component of 547 and $508 \mathrm{~nm} / \mathrm{mm}$, respectively. (b) The normalized intrinsic emittance increases with the laser beam size, contradicting Eq. (1) and is explained by the gun design. explanation. The nonlinear dependence of the core slice emittance due to the asymmetric field in the gun was verified by laser beam size scans at reduced gun gradient where, as expected from the rf-induced effect, the quadratic component was reduced.

Taking this quadratic component into account the normalized intrinsic emittance can be determined as the linear coefficient in the quadratic fit to the measurements. For the 260.1 and $267.6 \mathrm{~nm}$ laser wavelengths we determined normalized intrinsic emittances of $547 \pm 10$ and $508 \pm 35 \mathrm{~nm} / \mathrm{mm}$, respectively. This nonlinearity means that measurements performed with only the smallest aperture systematically overestimate the intrinsic emittance by $10 \%-20 \%$.

\section{Effective work function measurements \\ 1. $Q E$ as a function of laser wavelength}

To estimate the intrinsic emittance from the effective work function, the QE was measured for each wavelength by recording the charge at a calibrated beam position monitor downstream of the gun $(\sim 2.6 \mathrm{~m})$, while changing the laser energy (Fig. 4). The measurements were taken with the Ti:sapphire system as well as the OPA, covering a total wavelength range of $248-301 \mathrm{~nm}$. The inset shows the best fit found for $\mathrm{Cu}_{3} 3$ with an effective work function of $3.93 \pm 0.03 \mathrm{eV}$, using the dependence described in Eq. (4).

\section{QE as a function of injection phase}

The charge was also measured as a function of the injection phase of the laser relative to the gun rf (Schottky scan) to vary the magnitude of the Schottky effect (Fig. 5).

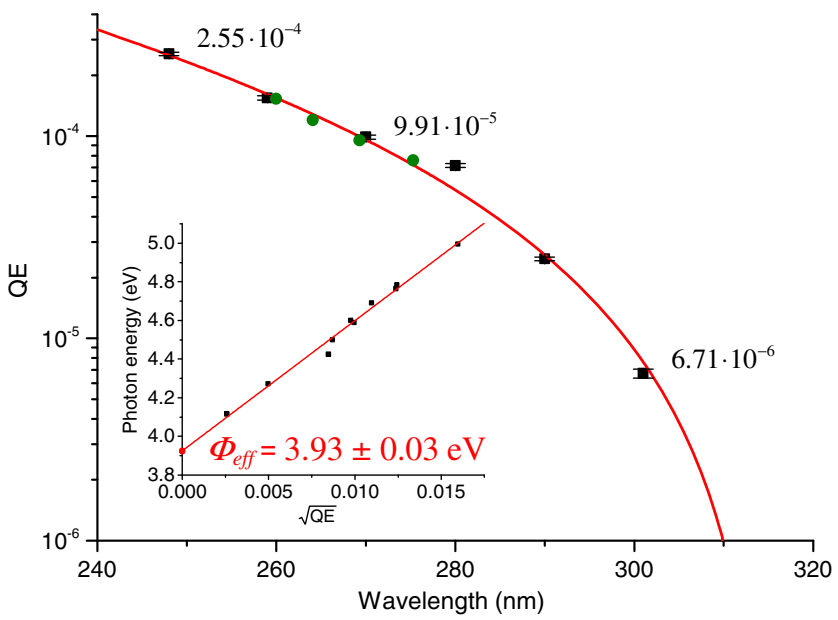

FIG. 4. Quantum efficiency as a function of wavelength with $\mathrm{Cu} 3$ cathode. Numbers are shown at the two extremes and at the nominal wavelength $(248,270$, and $301 \mathrm{~nm})$. Measurements were taken with both the 3rd harmonic of the Ti:sapphire laser 3rd harmonic (green dots) and the OPA (black squares) covering a total wavelength range of 248-301 nm. The fit based on Eq. (4) (inset) gives an effective work function of $3.93 \mathrm{eV}$ (red line). 


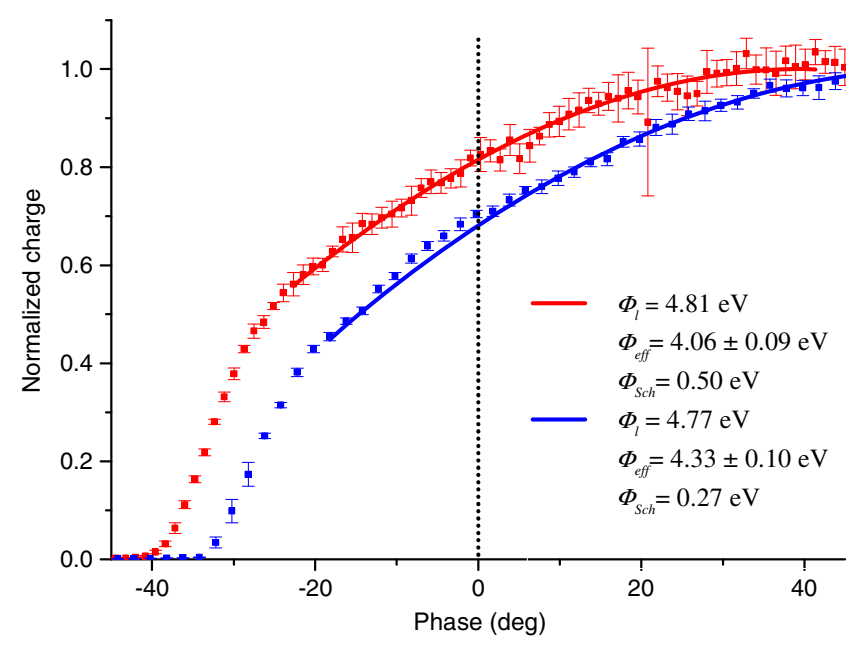

FIG. 5. Charge as a function of injection phase at $257.5 \mathrm{~nm}$ laser wavelength (red points) to determine effective work function and at $260 \mathrm{~nm}$ a month earlier (blue points). Best fit (solid red line) results in a work function of $4.56 \mathrm{eV}$ and a field enhancement factor of 4.18, corresponding to $0.50 \mathrm{eV}$ Schottky effect, while for the second case (blue line) a work function of $4.60 \mathrm{eV}$ and a field enhancement factor of 1, corresponding to $0.27 \mathrm{eV}$ Schottky effect. Zero degree is the operational phase for the intrinsic emittance measurements.

By using Eqs. (2)-(4) and fitting to the points around the operational phase (to guarantee a full transmission of the extracted charge) the best fit is found with a work function of $4.56 \mathrm{eV}$ for this cathode, giving an effective work function of $4.06 \pm 0.09 \mathrm{eV}$. This value is equivalent with the one obtained from the wavelength scans $(3.93 \pm 0.03 \mathrm{eV})$.

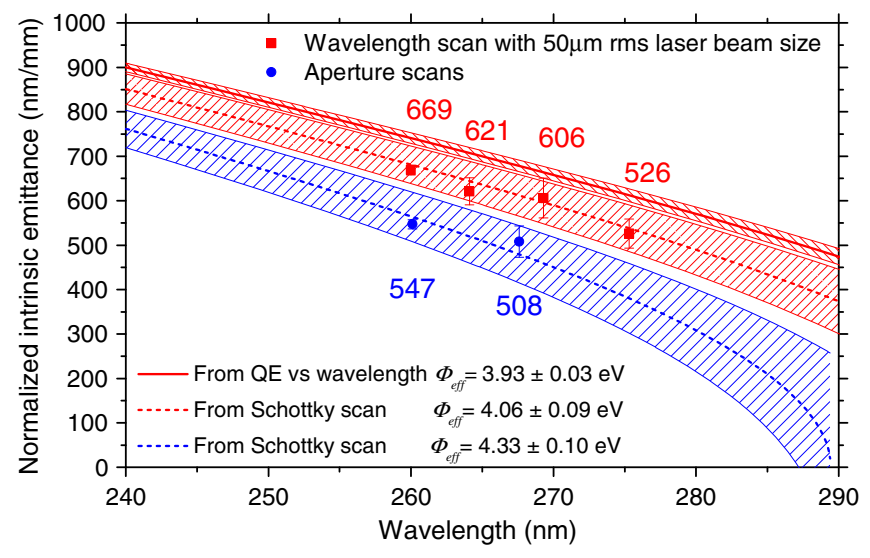

FIG. 6. Core slice emittance normalized to beam size as a function of wavelength for the $\mathrm{Cu}$ _ 3 cathode. Measurements with the smallest aperture below space-charge limit, at $1 \mathrm{pC}$ (red squares). Fits with intrinsic emittance calculated from the effective work functions determined from the QE measurements as a function of wavelength shown in Fig. 4 (solid red line with error band) and from the Schottky measurements (dashed red line with error band). Two points derived from the laser beam size scan a month earlier shown in Fig. 2 (blue dots) with the fit from the Schottky scan performed at the same time (dashed blue line with error band).

\section{Intrinsic emittance}

Figure 6 summarizes the intrinsic emittance measurements performed on the $\mathrm{Cu}_{\_} 3$ cathode with the $3 \mathrm{rd}$ harmonic of the Ti:sapphire laser. The two blue points are from the measurement shown in Fig. 3. One month later measurements were taken at four different wavelengths, represented by the red data points, ranging from 4.50 to $4.77 \mathrm{eV}$ photon energy. These four measurements were made at the smallest aperture, corresponding to $50 \mu \mathrm{m} \mathrm{rms}$ laser beam size on the cathode. The points were corrected taking into account the rf nonlinearity contribution, which we assume to be equal to the one of the measurements shown in Fig. 3.

The intrinsic emittance under the same laser and gun conditions degraded about $20 \%$ within a month, showing changes in the cathode properties. This can also be observed in the effective work functions derived from Schottky scans, providing a reliable estimate of the thermal emittance.

\section{CONCLUSIONS}

We have performed intrinsic emittance measurements with a copper cathode, while tuning the laser photon energy closer to the effective work function of the material, as suggested by Ref. [20].

An accurate estimation of the normalized intrinsic emittance is not possible from a single QE measurement. Therefore we have determined the effective work function by scanning the laser wavelength and the gun field, while measuring the emitted charge. The estimated values for the intrinsic emittance, especially the ones obtained from the Schottky scans, agree with the core slice emittance measurements performed at low charge and small laser aperture size. Work function and intrinsic emittance must be measured experimentally for each cathode within a short time interval to allow a reasonable matching with the theory. Schottky scans are a fast and reliable tool that can be used to select the cathode for optimal operation and to monitor its quality over time.

Special care was taken to perform core slice emittance measurements in the space-charge-free regime. The lowest normalized intrinsic emittance of $508 \mathrm{~nm} / \mathrm{mm}$ was reached with the $\mathrm{Cu}_{-} 3$ cathode at $267.6 \mathrm{~nm}$ and $49.4 \mathrm{MV} / \mathrm{m}$ field on the cathode, which is in good agreement with the theory. The lowest intrinsic emittance measured with $50 \mu \mathrm{m}$ rms laser beam size on the cathode was $30 \mathrm{~nm}$ at $1 \mathrm{pC}$.

We furthermore have shown that using a tunable Ti: sapphire laser source allows a reduction of intrinsic emittance, if sufficient laser energy is available to compensate for the lower QE. The reduction of emittance within the $20 \mathrm{~nm}$ wavelength range explored here is $\sim 20 \%$, which gives a $20 \%$ increase in the FEL output power at $200 \mathrm{pC}$ bunch charge. 


\section{ACKNOWLEDGMENTS}

We thank R. Ischebeck and his group for the development of the low charge emittance monitor, B. Beutner for his contribution to the data analysis software, L. Stingelin and R. Ganter for the cathode preparation, F. Le Pimpec for useful discussions regarding cathode properties, and $\mathrm{M}$. Pedrozzi for careful proofreading of the manuscript.

[1] N. Rohringer, D. Ryan, R. A. London, M. Purvis, F. Albert, J. Dunn, J. D. Bozek, C. Bostedt, A. Graf, R. Hill, S. P. Hau-Riege, and J. J. Rocca, Nature (London) 481, 488 (2012).

[2] H. N. Chapman, P. Fromme, A. Barty, T. A. White, R. A. Kirian, A. Aquila, M. S. Hunter, J. Schulz, D. P. DePonte et al., Nature (London) 470, 73 (2011).

[3] L. Young et al., Nature (London) 466, 56 (2010).

[4] A. Fratalocchi and G. Ruocco, Phys. Rev. Lett. 106, 105504 (2011).

[5] H. N. Chapman et al., Nat. Phys. 2, 839 (2006).

[6] Y. Ding et al., Phys. Rev. Lett. 102, 254801 (2009).

[7] M. Krasilnikov et al., Phys. Rev. ST Accel. Beams 15, 100701 (2012).

[8] E. Prat, M. Aiba, S. Bettoni, B. Beutner, S. Reiche, and T. Schietinger, Phys. Rev. ST Accel. Beams 17, 104401 (2014).

[9] F. Zhou, A. Brachmann, P. Emma, S. Gilevich, and Z. Huang, Phys. Rev. ST Accel. Beams 15, 090701 (2012).

[10] SwissFEL Conceptual Design Report, edited by R. Ganter, PSI Report No. 10-04, 2011.

[11] S. Bettoni, M. Pedrozzi, and S. Reiche, in Proceedings of 35th International Free-Electron Laser Conference (JACoW, Manhattan, USA, 2013), p. 219, TUPSO07.

[12] K. Flöttmann, ASTRA user manual, www.desy.de/ mpyflo/Astra_dokumentation.

[13] S. Reiche, Nucl. Instrum. Methods Phys. Res., Sect. A 429, 243 (1999).

[14] J. B. Rosenzweig et al., Nucl. Instrum. Methods Phys. Res., Sect. A 593, 39 (2008).

[15] S. Reiche, P. C. Pellegrini, and J. B. Rosenzweig, Nucl. Instrum. Methods Phys. Res., Sect. A 593, 45 (2008).

[16] J. Yang, F. Sakai, T. Yanagida, M. Yorozu, Y. Okada, K. Takasago, A. Endo, A. Yada, and M. Washio, J. Appl. Phys. 92, 1608 (2002)

[17] S. Bettoni, M. Pedrozzi, S. Reiche, and T. Schietinger, in Proceedings of the 2nd International Particle Accelerator Conference, San Sebastián, Spain (EPS-AG, Spain, 2011), p. 3107, THPC093.
[18] A. Cianchi et al., Phys. Rev. ST Accel. Beams 11, 032801 (2008).

[19] SwissFEL Injector Conceptual Design Report, edited by M. Pedrozzi, PSI Report No. 10-05, 2010.

[20] D. H. Dowell and J. F. Schmerge, Phys. Rev. ST Accel. Beams 12, 074201 (2009).

[21] C. P. Hauri, R. Ganter, F. Le Pimpec, A. Trisorio, C. Ruchert, and H.H. Braun, Phys. Rev. Lett. 104, 234802 (2010).

[22] H. J. Qian, C. Li, Y. C. Du, L. X. Yan, J. F. Hua, W. H. Huang, and C. X. Tang, Phys. Rev. ST Accel. Beams 15, 040102 (2012).

[23] Z. M. Yusof, M. E. Conde, and W. Gai, Phys. Rev. Lett. 93, 114801 (2004).

[24] Y. Huang and Y. Miyahara, Simulation on the Emittance of the RF Gun Including the Schottky Effect, in Proceedings of the 18th Linear Accelerator Conference (CERN, Geneva, Switzerland, 1996), p. 87, THP84.

[25] H. Chen, Y. Du, W. Gai, A. Grudiev, J. Hua, W. Huang, J. G. Power, E. E. Wisniewski, W. Wuensch, C. Tang, L. Yan, and Y. You, Phys. Rev. Lett. 109, 204802 (2012).

[26] M. Krasilnikov, Impact of the Cathode Roughness on the Emittance of an Electron Beam, in Proceedings of the 28th Free Electron Laser Conference (JACoW, Berlin, Germany, 2006), p. 583, THPPH013, https://accelconf.web .cern.ch/accelconf/f06/PAPERS/THPPH013.PDF.

[27] T. Munakata, T. Sugiyama, T. Masuda, M. Aida, and N. Ueno, Appl. Phys. Lett. 85, 3584 (2004).

[28] CRC Handbook of Chemistry and Physics 1st Student Edition, edited by R. C. Weast (CRC Press, Boca Raton, FL, 1988), E-78.

[29] J. F. Schmerge, J. M. Castro, J. E. Clendenin, D. H. Dowell, S. M. Gierman, and R.O. Hettel, in Proceedings of FEL2004 Conference (Comitato Conferenze Elettra, Trieste, Italy, 2004), p. 205.

[30] R. Bossart and M. Dehler, Design of a RF Gun for Heavy Beam Loading, in Proceedings of the 5th European Particle Accelerator Conference (Insitute of Physics Publishing, Sitges, Spain, 1996), p. 1544, THP013L, http:// accelconf.web.cern.ch/AccelConf/e96/PAPERS/THPL/ THP013L.PDF.

[31] A. Trisorio, P. M. Paul, F. Ple, C. Ruchert, C. Vicario, and C. P. Hauri, Opt. Express 19, 20128 (2011).

[32] Light Conversion, http://www.lightcon.com/, Keramikų 2, Vilnius, LT-10233, Lithuania.

[33] R. Ischebeck and V. Thominet, Patent No. WO2014029693A1, 2014.

[34] L. Xiao, R. F. Boyce, D. H. Dowell, Z. Li, C. LimborgDeprey, and J.F. Schmerge, in Proceedings of the 21st Particle Accelerator Conference, Knoxville, TN, 2005 (IEEE, Piscataway, NJ, 2005), p. 3432, TPPE058. 